\title{
Paraganglioma of the Urinary Bladder: A Case Report
}

\author{
Yara A. Fayoumi ', MBBS, Sarah A. Almagharbi ${ }^{1}$, MBBS, \\ Taha A. Abdel-Meguid ${ }^{2,3}$, MBBS, MSc, Ahmed Abdelsalam ${ }^{2,4}$, MD, MSc, \\ Najla M. Alghanmi ${ }^{5}$, MBBCh, KSFP, and Haneen Al-Maghrabi ${ }^{6}$, MD, $^{2}$ \\ Raed A. Azhar ${ }^{2,7}$, FACS, FRCSC \\ ${ }^{1}$ Faculty of Medicine, King Abdulaziz University, Jeddah, Saudi Arabia \\ ${ }^{2}$ Department of Urology, Faculty of Medicine, King Abdulaziz University, Jeddah, Saudi Arabia \\ ${ }^{3}$ Department of Urology, Faculty of Medicine, Minia University, Minia, Egypt \\ ${ }^{4}$ Theodor Bilharz Research Institute, Giza, Egypt \\ ${ }^{5}$ Department of Pathology, King Abdulaziz University Hospital, Jeddah, Saudi Arabia \\ ${ }^{6}$ Department of Pathology, King Faisal Specialist Hospital and Research Center, Jeddah, Saudi Arabia \\ 'UC Institute of Urology, Keck School of Medicine, University of Southern California, Los Angeles, CA USA
}

\section{Correspondence \\ Dr. Raed A. Azhar \\ Department of Urology, Faculty of Medicine \\ King Abdulaziz University \\ P.O. Box 80215, Jeddah 21589 \\ Saudi Arabia \\ e.M: raazhar@kau.edu.sa \\ Submission: 11 Sept. 2018 \\ Accepted: 19 Nov. 2018 \\ Citation \\ Fayoumi YA, Almagharbi SA, Abdel-Maguid TA, Abdelsalam A, AlGhanmi NM, Al-Maghrabi H, Azhar RA. Paraganglioma of the urinary bladder: a case Report. JKAU Med Sci 2018; 25 (2): 57-62. DOI: 10.4197/Med. 25.2.7}

Copyright: $\odot$ The Author(s), YEAR. Publisher. The Journal of King Abdualziz University - Medical Sciences is an Official Publication of "King Abdulaziz University". It is an open-access article distributed "King Abdulaziz University". It is an open-access article distributed
under the terms of the Creative Commons Attribution Non-Commercial License, which permit unrestricted non-commercial use, distribution, and reproduction in any medium, provided the original work is properly cited.

\begin{abstract}
Urinary bladder paragangliomas are rare with a reported incidence of $<1 \%$ of bladder tumors. We presented a case of a urinary bladder paraganglioma in a 26-year-old male who presented with a painless gross hematuria and hypertensive crisis occurring during his transurethral resection. Our diagnosis was based upon patient's history, abdominal-pelvic computed tomography scan, which revealed a left sided bladder wall mass $2.26 \mathrm{X} 4.4 \times 3.28 \mathrm{~cm}$ that was obstructing the left distal ureter and causing significant left severe hydroureter and hydronephrosis. Preoperative preparation was done with beta blocker and alpha blocker two weeks prior to surgery. The patient underwent an open surgical partial cystectomy, left nephroureterectomy and, complete external and internal iliac lymphadenectomy. Gross pathological examination of the mass revealed a $3 \times 4.5 \times 4 \mathrm{~cm}$ growth originating from the wall of urinary bladder. Microscopic examination revealed nests invaded into muscularis propria of the urinary bladder, with no evidence of distant metastasis. Immunohistochemical studies showed positive tumor cells staining for chromogranin and synaptophysin while S100 highlighted the surrounding sustentacular cells. During the first 3-month post-operative follow-up period, the patient's blood pressure was stabilized and the symptoms gradually subsided. Urinary bladder paragangliomas should be considered among the differential diagnosis of bladder tumors..
\end{abstract}

\section{Keywords}

Paraganglioma; Urinary bladder; Pheochromocytoma; Transurethral resection of prostate 


\section{Introduction}

$P$ heochromocytomas are catecholamine secreting tumors that originate from chromaffin cells of the adrenal medulla and the sympathetic ganglia. Around $10 \%$ of pheochromocytomas arise from extra-adrenal organs and are termed paragangliomas. Primary bladder pheochromocytoma is a rare neoplasm accounting for $0.06 \%$ of all bladder tumors ${ }^{[1]}$. These tumors tend to be functional, i.e., secreting catecholamine which causes palpitations, hypertension and micturition syncope $^{[2]}$. Classic clinical presentation reported in the literature include hematuria and symptoms related to catecholamine overload induced by micturition or defecation such as paroxysmal hypertension, palpitations and attacks of micturition headache ${ }^{[3]}$. Computed tomography (CT) scan and magnetic resonance imaging (MRI) could provide a comprehensive characteristic for the diagnosis of paragangliomas and pheochromocytomas ${ }^{[3]}$. Benign and malignant pheochromocytomas are histologically and biochemically similar ${ }^{[4]}$. However, immunohistochemistry is the only definitive modality used to diagnose paragangliomas and pheochromocytomas. Reported managements include radical cystectomy and partial cystectomy by transurethral approach.

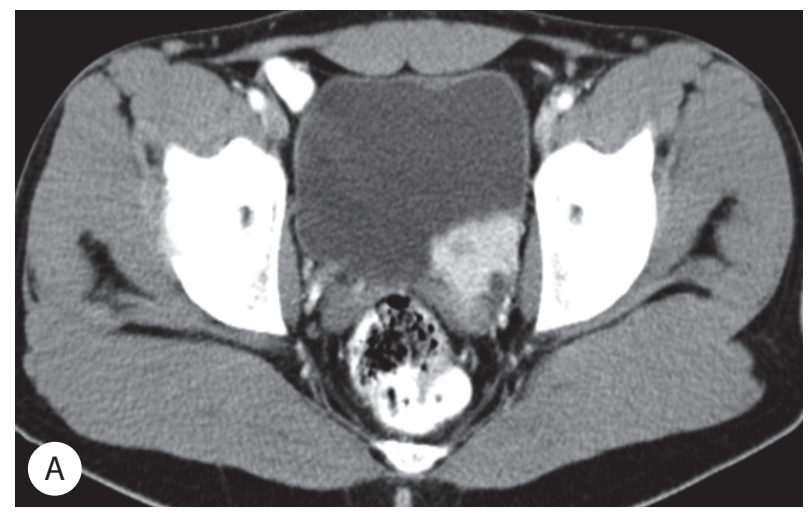

Figure 1. A-B. There is a heterogeneous enhancing soft tissue mass arising from the left superior lateral wall of the bladder, region measure $2.26 \times 4.4 \times 3.28$ in anteroposterior (AP), craniocaudal plan respectively. The mass is enhancing the left distal ureter and uretervesical junction. The mass is also infiltrating the left seminal vesical. There are two enlarged left external iliac lymph nodes showing hypervascularity with the largest one measuring $2.1 \times 2.3$ $\mathrm{cm}$ and the smaller measuring $1.5 \times 1.6 \mathrm{~cm}$.

\section{Case Report}

A 26-year-old heavy smoker Yemeni male, who was otherwise healthy, had presented to another hospital in October 2016 with painless gross hematuria with blood clots. The patient also reported episodic headaches, sweating, palpitations, tremors, nausea and vomiting after voiding. Findings on physical examination were unremarkable. Cystoscopy was done there which revealed bladder tumor. Transurethral resection of bladder tumors (TURBT) was preformed but the procedure was complicated by hypertensive crisis which required ICU admission.

The patient was then referred to our hospital with a report of TURBT specimen showing a bladder tumor infiltrating the muscularis propria.

In our hospital, abdominal-pelvic CT scan revealed a left sided bladder wall mas $2.26 \times 4.4 \times 3.28 \mathrm{~cm}$ that was obstructing the left distal ureter and causing significant left severe hydroureter and hydronephrosis with a very thin atrophic parenchyma. Two left iliac lymphadenopathies were also demonstrated with no evidence of distant metastasis (Fig. 1).

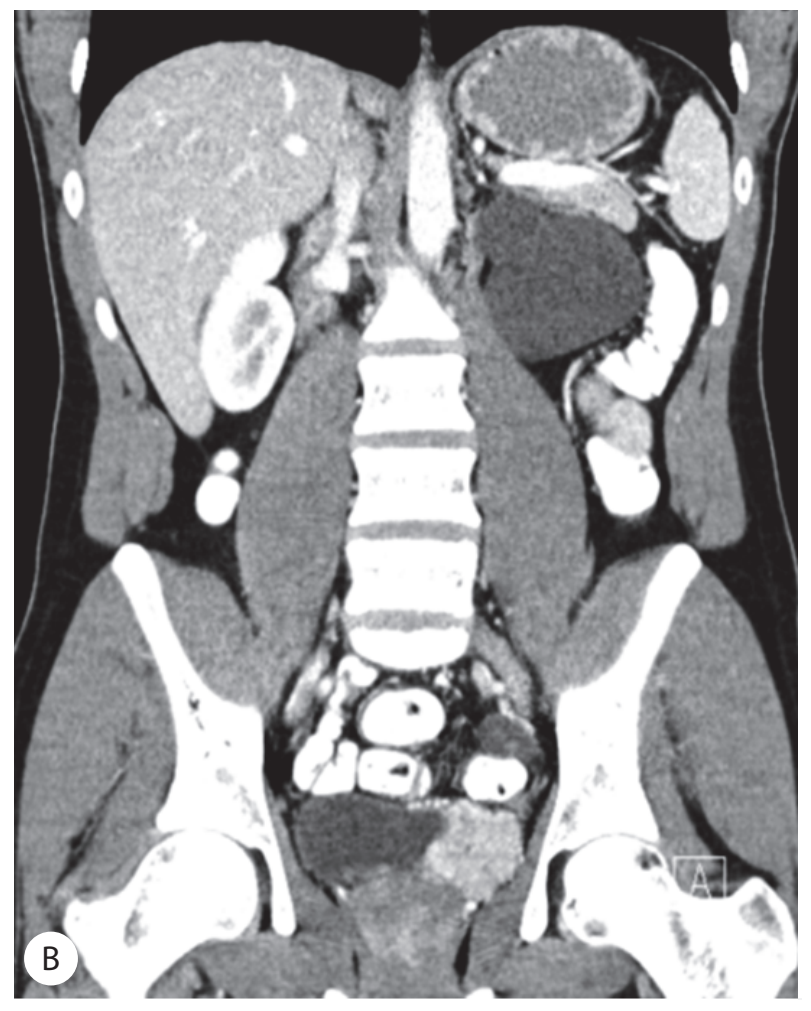


Preoperative preparation was done with beta blocker and alpha blocker two weeks prior to surgery. The patient underwent an open surgical partial cystectomy, left nephroureterectomy and, complete external and internal iliac lymphadenectomy. Frozen sections examination demonstrated surgical margins that were negative for malignancy. The patient was discharged on good condition with a Foley's catheter.

Gross pathologic examination of the mass revealed a $3 \times 4.5 \times 4 \mathrm{~cm}$, dark brownish, rounded and firm growth originating from the wall of urinary bladder.

Microscopic examination of the tumor revealed nests of cells containing moderate to large amount of eosinophilic cytoplasm (Fig. 2A, 2B). These nests were surrounded by a thin network of fibrovascular tissue forming "zellballen" pattern (Fig. 2C). Infrequent mitotic activity was noted. The tumor nests invaded into muscularis propria of the urinary bladder (Fig. 2.D). No lymphovascular or perineural invasion were detected. Sections from the left external iliac lymph nodes revealed metastatic tumor deposits. Immunohistochemical studies showed positive tumor cells staining for chromogranin and synaptophysin while S100 highlighted the surrounding sustentacular cells (Fig. 3).

During the first 3-month post-operative follow-up period, the patient's blood pressure was stabilized and the symptoms gradually subsided.

\section{Discussion}

Pheochromocytomas are originated from the adrenal gland, while paragangliomas are extra-adrenal pheochromocytomas which account for 15-20\% of all pheochromocytoma ${ }^{[5]}$. Bladder paragangliomas are unusual neoplasms, accounting for less than $1 \%$ of all pheochromocytomas with a malignant potential of $10 \%$. They are hormonally active with raised catecholamine metabolites release ${ }^{[6]}$.
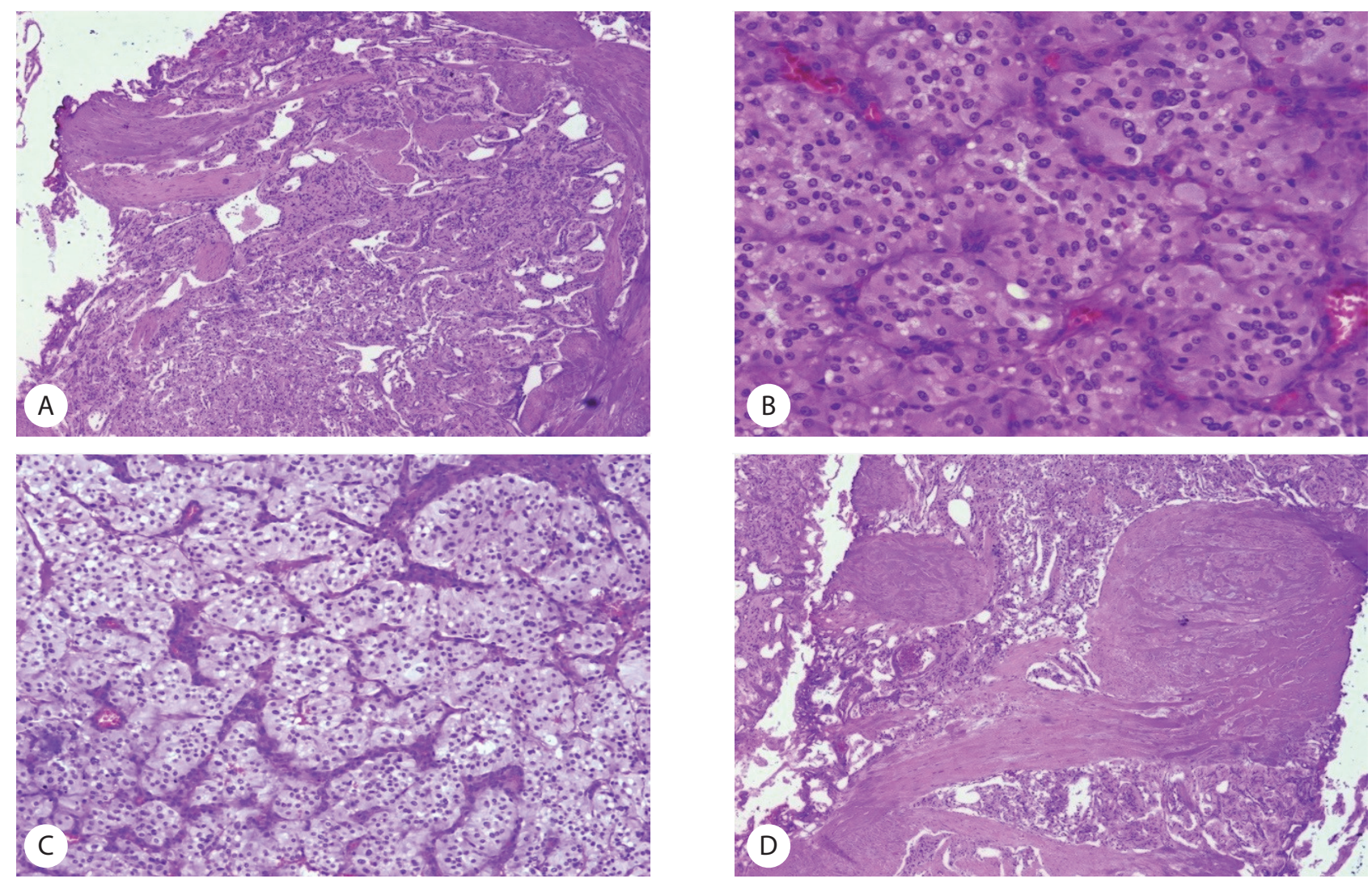

Figure 2. A, B, C, D. Histopathology of paraganglioma (Hematoxylin and Eosin Stain). A: Infiltrative tumor cells arranged in nests (H\&E Stain; magnification $x 4$ ). B: Tumor nests invade into muscularis propria (H\&E Stain; magnification x4). C: Tumor nests arranged in the characteristic zellballen patter (H\&E Stain; magnification $\times 10)$. D: Plump tumor cells with eosinophilic to amphophilic type of cytoplasm showing low mitotic activity (H\&E Stain; magnification x20). 

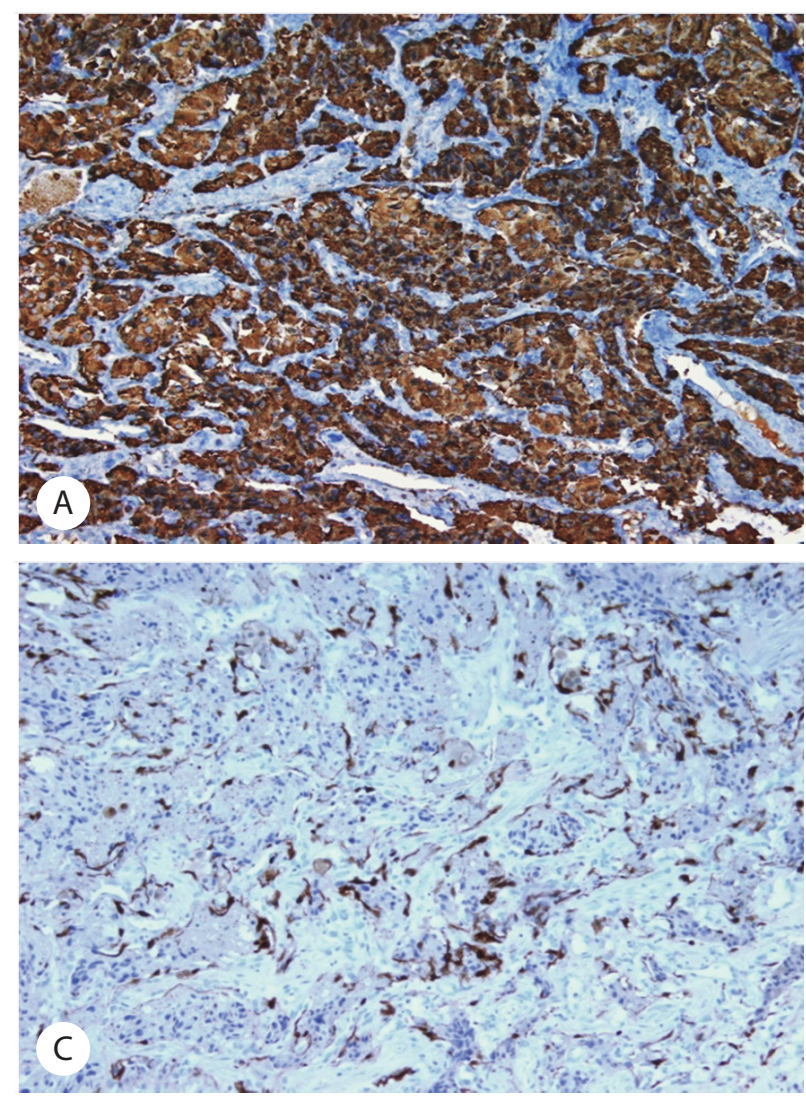

Urinary bladder pheochromocytomas (paragangliomas) are more predominant in middle aged adults with females more frequently affected. These tumors commonly present with painless gross hematuria, hypertension along with symptoms of elevated catecholamines such as headache, palpitations, blurred vision, and flushing ${ }^{[7]}$. Our patient had presented with hematuria, headache and hypertension. Radiological CT of abdomen and pelvic are sensitive in detecting $94 \%$ of adrenal and extra-adrenal pheochromocytomas, however, MRI is much more sensitive for detecting small extra-adrenal pheochromocytomas ${ }^{[8]}$. Cystoscopic examination can define tumor's location, invasion depth and ureteric involvement.

In our case, microscopically, the "zellballen" pattern of nests were seen invading the muscularis propria of the urinary bladder. The tumor was invading the perivesicular tissue, and involving three iliac lymph nodes. Immunohistochemistry analysis showed a positive reaction of tumor cells to chromografin and synaptophysin, focally positive S-100 and negative for panCK. The most well-known pathological finding is

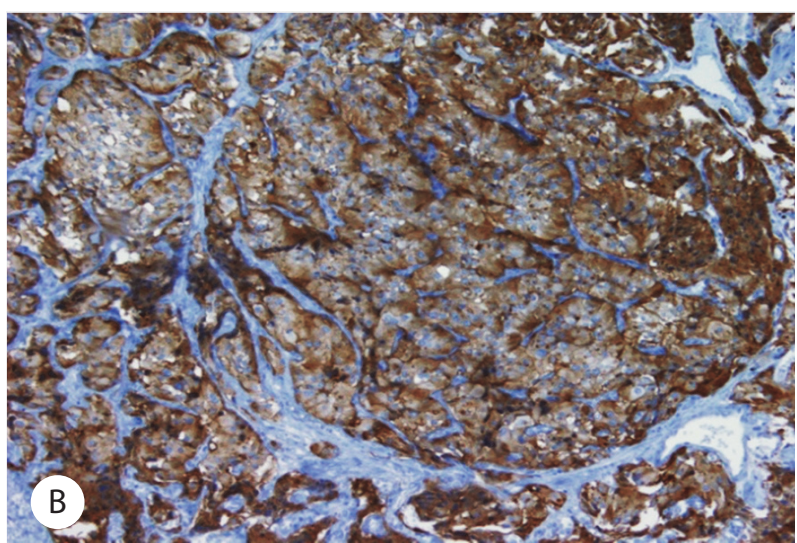

Figure 3. A, B, C. Immunohistochemical studies of paraganglioma. A:Tumor cells are positive for chromogranin (Immunohistochemistry stain; magnification $\mathrm{x} 10$ ) and B: synaptophysin (Immunohistochemistry stain; magnification $\mathrm{x10}$ ), while C: S100 highlights sustentactular cells in between (Immunohistochemistry stain; magnification $\times 10$ ).

"Zellballen" pattern, in which immunohistochemical staining is usually positive for synaptophysin and chromogranin, with $\mathrm{S}-100$ being highlighted in sustentacular cells ${ }^{[9]}$.

Based on these findings, a diagnosis of extra adrenal paraganglionic pheochromocytoma was established. Although urinary bladder malignant paragangliomas are considered extremely rare, clinicians need to consider it in their differential diagnosis of bladder neoplasms especially in patients demonstrating secondary complications during tumor manipulation or during resection. Generally, malignant paragangliomas of the urinary bladder arise more commonly in males, young to middle aged. The most common presentation is with signs and symptoms of catecholamine crisis. Interestingly, the size does not usually correlate with possibility of metastasis; mostly metastases to the regional lymph nodes.

Transurethral resection, laparoscopic or open cystectomy (partial or radical) are the reported treatment for bladder paragangliomas with the aim of negative resection margins ${ }^{[9]}$. It is recommended to be very cautious intraoperatively with functioning tumors in order to prevent hypertensive crises. The role of adjuvant therapy is indicated in unrespectable lesions, advanced cases and in metastatic disease ${ }^{[10,11]}$. 
Around $3 \%$ of patients with reported follow-up died due to their cancer, illuminating that good survival rates can be accomplished by the above therapies. It is important to point out that around $20 \%$ of patients had recurrence or metastases at follow-ups ${ }^{[12,13]}$. Patients with localized tumors have a promising prognosis and may be managed by less radical modalities, with a significantly high survival rate.

Moving forward, it would be helpful to standardize the reporting guidelines of paragangliomas cases for better understanding of the natural process and outcomes $^{[14]}$.

\section{Conclusion}

In summary, we reported a rare case of 26-year-old male with a functional paraganglioma of the urinary bladder, who presented with painless gross hematuria and catecholamine releasing symptoms. After proper preoperative preparation, the patient underwent partial cystectomy, left nephroureterectomy and, complete external and internal iliac lymphadenectomy with documented tumor-free surgical margins. At follow up, the hypertension was stabilized and the symptoms gradually subsided.

Even though urinary bladder malignant paragangliomas are extremely rare neoplasms, they should be considered among the differential diagnosis of bladder tumors, particularly in cases with suspected functional tumors.

\section{Conflict of Interest}

The authors have no conflict of interest.

\section{Disclosure}

The authors did not receive any type of commercial support either in forms of compensation or financial for this study. The author has no financial interest in any of the products or devices, or drugs mentioned in this article.

\section{Ethical Approval}

Obtained.

\section{References}

[1] Melicow MM. One hundred cases of pheochromocytoma (107 tumors) at the Columbia-Presbyterian Medical Center 1926-1976: a clinicopathological analysis. Cancer 1977; 40(5): 1987-2004.

[2] Sheps SG, Jiang NS, Klee GG, van Heerden JA. Recent developments in the diagnosis and treatment of pheochromocytoma. Mayo Clin Proc 1990; 65(1): 88-95.

[3] Vahidi K, Joe BN, Meng M, Coakley FV, Yeh BM. Review of atypical pelvic masses on CT and MRI: expanding the differential diagnosis. Clin Imaging 2007; 31(6): 406-413.

[4] Geng JH, Huang SP, Shen JT, Chend YT. Paraganglioma of the urinary bladder with pelvic metastasis. Urological Sci 2014; 25(3): 91-94.

[5] Erickson D, Kudva YC, Ebersold MJ, Thompson GB, Grant CS, van Heerden JA, Young WF Jr. Clin Endocrinol Metab 2001; 86(11): 5210-5216.

[6] Naqiyah I, Rohaizak M, Meah FA, Nazri MJ, Sundram M, Amram AR. Phaeochromocytoma of the urinary bladder. Singapore Med J 2005; 46(7): 344-346.

[7] Siatelis A, Konstantinidis C, Volanis D, Leontara V, ThomaTsagli E, Delakas D.. Pheochromocytoma of the urinary bladder: report of 2 cases and review of literature. Minerva Urol Nefrol 2008; 60(2): 137-140.

[8] Gittes RF, Mahoney EM. Pheochromocytoma. Urol Clin North Am 1977; 4(2): 239-252.

[9] Doran F, Varinli S, Bayazit Y, Bal N, Ozdemir S. Pheochromocytoma of the urinary bladder. APMIS 2002; 110(10): 733-736.

[10] Whalen RK, Althausen AF, Daniels GH. Extra-adrenal pheochromocytoma. J Urol 1992; 147(1): 1-10.

[11] Zwahlen D, Fishman PN, Honey J, Milosevic M, Tannock I. Malignant pheochromocytoma of the urinary bladder. Can J Urol 2007; 14(1): 3455-3457.

[12] Jansen R, Zaslau S. Paraganglioma of the bladder. Clin Adv Hematol Oncol 2012; 10(12): 839-841.

[13] Tsai CC, Wu WJ, Chueh KS, Li WM, Huang CH, Wu CC, Lee $\mathrm{MH}$, Chen SM. Paraganglioma of the urinary bladder first presented by bladder bloody tamponade: two case reports and review of the literatures. Kaohsiung J Med Sci 2011; 27(3): 108-113.

[14] Beilan J, Lawton A, Hajdenberg J, Rosser CJ. Locally advanced paraganglioma of the urinary bladder: a case report. BMC Res Notes 2013; 6: 156. 


\title{
ورم المستقتمات في المثانة ـ تقريز حالة
}

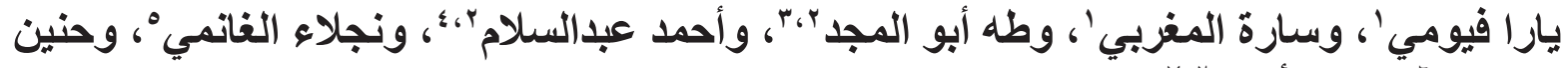

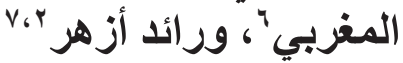

\author{
'كلية الطب، جامعة الملك عبدالعزيز ، جدة ـ المملكة العربية السعودية

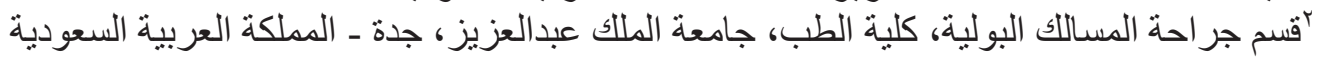

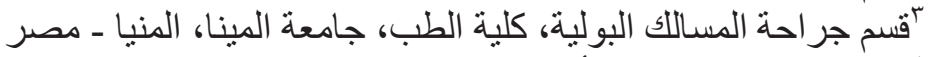

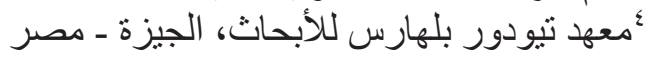

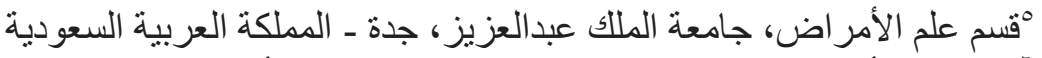

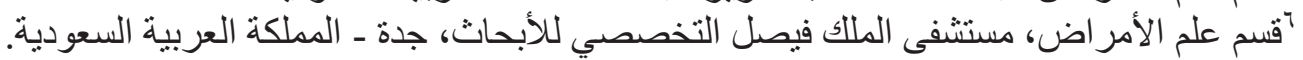

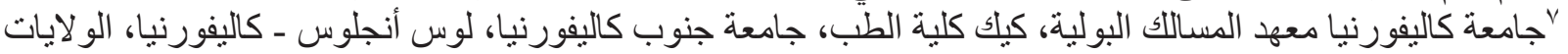 \\ المتحدة
}

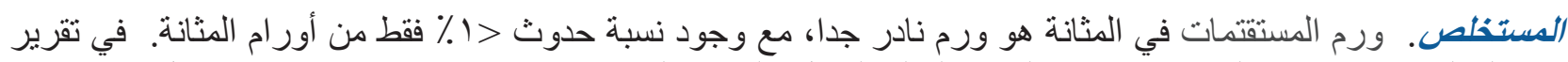

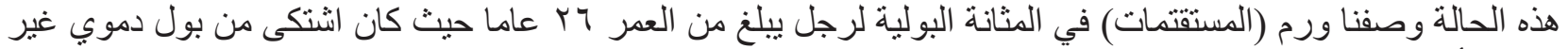

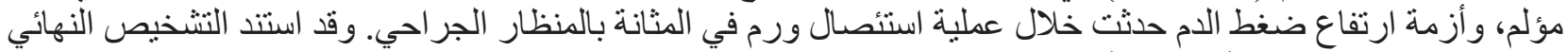

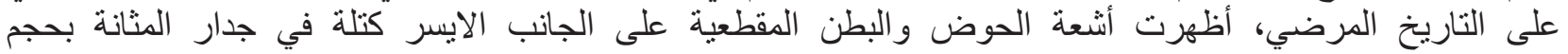

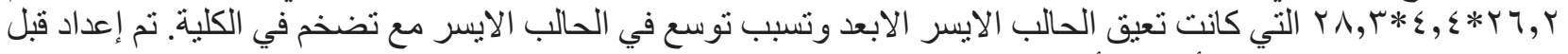

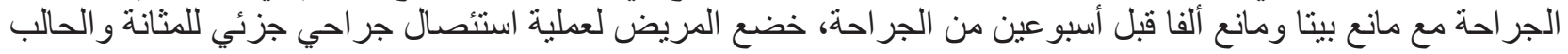

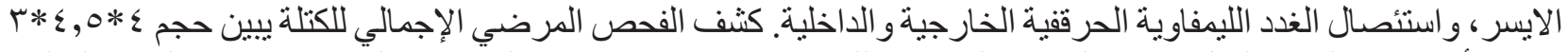

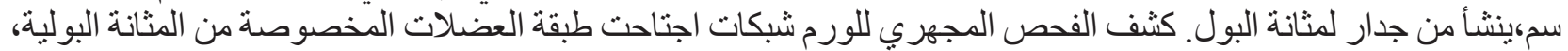

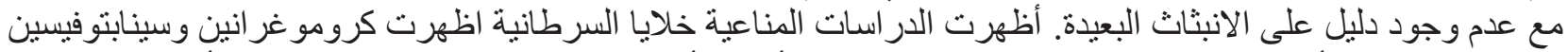

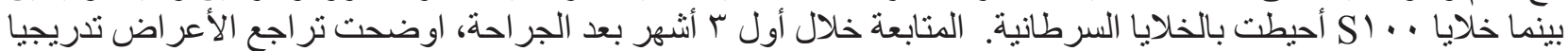

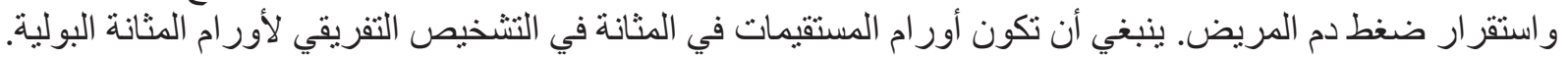

